\title{
Multi-objective traffic signal optimization using 3D mesoscopic simulation and evolutionary algorithms
}

\author{
Adriana Simona Mihăiţăa, ${ }^{a}$, Laurent Dupont ${ }^{\mathrm{b}}$, Mauricio Camargo ${ }^{\mathrm{b}}$ \\ ${ }^{a}$ Data61-CSIRO, 13 Garden St, Eveleigh, 2015, NSW, Australia \\ ${ }^{b}$ ERPI Laboratory EA6737, Lorraine University, 8 Rue Bastien Lepage, 54010 Nancy
}

\begin{abstract}
Modern cities are currently facing rapid urban growth and struggle to maintain a sustainable development. In this context, "eco-neighbourhoods" became the perfect place for testing new innovative ideas that would reduce congestion and optimize traffic flow. The main motivation of this work is a true and stated need of the Department of Transport in Nancy, France, to improve the traffic flow in a central eco-neighbourhood currently under reconfiguration, reduce travel times and test various traffic control scenarios for a better interconnectivity between urban intersections. Therefore, this paper addresses a multi-objective simulation-based signal control problem through the case study of "Nancy Grand Cœur" (NGC) eco-neighbourhood with the purpose of finding the optimal traffic control plan to reduce congestion during peak hours. Firstly, we build the 3D mesoscopic simulation model of the most circulated intersection (C129) based on specifications from the traffic management centre. The simulation outputs from various scenario testing will be then used as inputs for the optimisation and comparative analysis modules. Secondly, we propose a multi-objective optimization method by using evolutionary algorithms and find the optimal traffic control plan to be used in C129 during morning and evening rush hours. Lastly, we take a more global view and extend the 3D simulation model to three other interconnected intersections, in order to analyse the impact of local optimisation on the surrounding traffic conditions in the econeighbourhood. The current proposed simulation-optimisation framework aims at supporting the traffic engineering decision-making process and the smart city dynamic by favouring a sustainable mobility.
\end{abstract}

Keywords: simulation-based transport planning, mesoscopic traffic simulation; multi-objective optimization; evolutionary algorithms; eco-neighbourhoods; smart city.

\footnotetext{
* Corresponding author

Email address: simona.mihaita@data61.csiro.au (Adriana Simona Mihăiţă)
} 


\section{Introduction}

Context: Urban areas have known a rapid growth during the last century. If in the 1950 s, only $30 \%$ of the population lived in cities, recent statistics show that this figure has reached $54 \%$ in 2014 , and could even reach $66 \%$ by 2050

5 1]. With this rapid increase of urban inhabitants, new problems have emerged, such as high congestion, low traffic fluidity, unsynchronized inter-modal transport systems, air pollution, noise, etc. Transportation plays a very important role in the lives of its citizens, as it provides access to jobs, health services, education and leisure. In 2008, in the European Union (EU), the total external

10 transport costs were estimated at over 500 billion Euro a year, which represented almost $4 \%$ of the gross domestic product (GDP) [2]. To these costs is added the annual cost of congestion, which amounts between 146 and 243 billion Euro, representing $1-2 \%$ of the GDP [3]. Given also the fact that Europe is a net importer of fossil fuels, to the tune of 388 billion Euro per year 4, a global ini-

15 tiative was adopted by EU to integrate together the concept of transportation, health and environment into a sustainable and healthy mobility.

In this context, the concept of eco-neighbourhood has emerged as a response to the above challenges, and became the perfect place for technical, innovative, economical and social experimentation. In 1960s, the first ecological areas "had

20 a rather small and remote location from metropolitan centres" [5]. Today, their role has become increasingly complex because they must meet several principles of sustainable development [6]: 1) involve all the citizens, 2) contribute to the improvement of daily life by developing a healthy and safe living environment, 3) participate in the economical and local dynamics, 4) promote a responsible 25 resource management and adapt to climate change. The eco-neighbourhoods offer the opportunity to experience and anticipate the city evolution, and guide the decision makers in their decision process. The latest changes in design practices (collaborative approach, usage integration directly from the design phase, citizen participation in experimental projects), offer new perspectives for simu-

so lating the impact of future urban reconfigurations [7. A detailed description of the evolution of European eco-neighbourhoods is provided in 8 .

Motivation With the urban project Nancy Grand Cœur [9] the Grand Nancy Metropolis in France, wants to rehabilitate the 15-hectares area around the historical train station including its railway and industrial brownfield. A

35 visual representation of the train station hosting almost 9 million passengers each year is provided in Figure 1 a). This ecological urban project is intended to be delivered by 2025, and the objectives for this central area are manifold: new fluid mobility, traffic regulation, reconciliation between historical and modern neighbourhoods of the city, environment quality and green spaces, reduced en-

40 ergy consumption, etc. As previously mentioned, improving traffic flow in this neighbourhood, reducing congestion and travel times represents a high priority for the traffic management center. A better mobility means reduced travel times, reduced pollution levels and improved connectivity between intersections.

Challenges: Our study focuses on congestion problems in four most circulated intersections of the NGC eco-neighbourhood (see selected area in sub-plots 


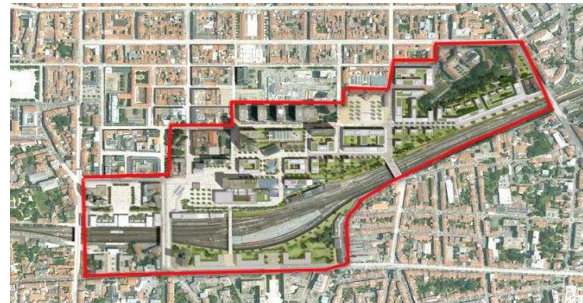

(a) Urban project for NGC by 2020 (Arep).

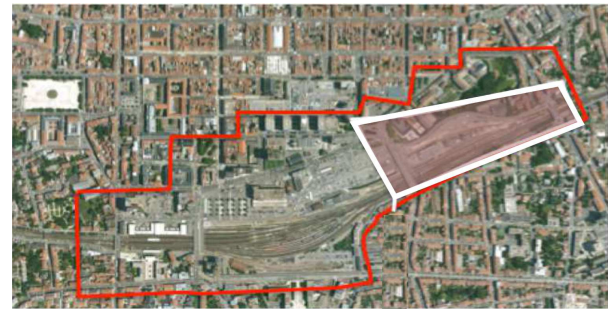

(b) Urban area in 2012 .

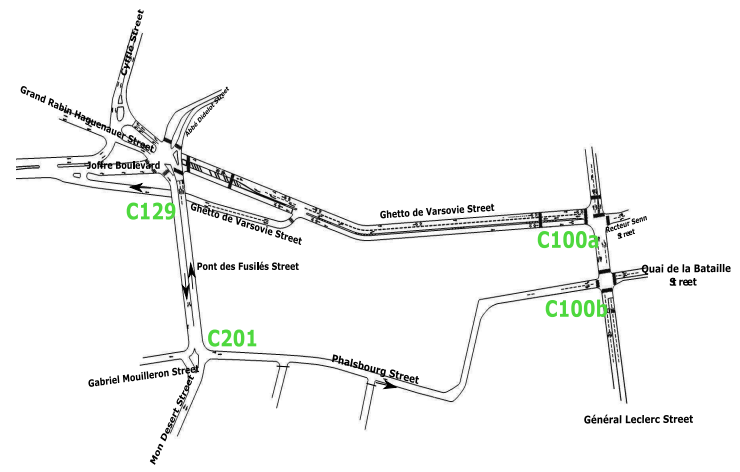

(c) Focus on the four most circulated intersections.

Figure 1: Case study of the eco-neighbourhood Nancy Grand Cœur.

b) and c) from Figure 11. As NGC project involves the reconfiguration and deviation of traffic flow from the most congested intersections to neighbouring free-flow areas, an important challenge is to find an adapted traffic signal plan for the most congested intersection $(\mathrm{C} 129)$ that would meet the following criteria simultaneously: decrease the average travel times when there is increased traffic flow during rush hours. By traffic signal plan we refer to the scheduling of red, orange and green lights during a fixed cycle-time interval. Therefore, we are facing a multi-objective constrained non-convex optimisation problem for which we propose a 3D simulation and evolutionary optimisation framework.

Solution: A first step to respond to this problem is to use adapted simulation tools, which will enable the analysis and testing of different traffic scenarios in NGC. We initially built a 3D mesoscopic traffic simulation model of the most circulated intersection in NGC (C129), which is one out of the main intersections connecting the train station to the Metropolis. This implies a high number of private vehicles and public transport lines crossing the intersection every day which have been integrated as well in the model. The model is initialised and validated using real data sets received from the local traffic management center. The second step is to address the optimization problem by turning to bio-inspired optimization methods, such as evolutionary algorithms. This part completes and improves our previous studies [10], as we extend the optimization 
process from a single to a multi-objective optimization procedure. The optimal traffic signal plan for C129 is then chosen by comparing the Pareto front of all the available traffic signal plans currently tested.

This paper is organized as follows. In Section 2 we present the state of the art on different traffic simulation tools and optimization methods. Our proposed optimization framework is provided in Section 3, while the construction of the 3D mesoscopic traffic simulation model is presented in Section 4 . In Section 4.2 we extend the 3D mesoscopic simulation model of C129 to three other adjoining intersections (C129, C201, C100a, C100b) as represented in Figure

75 1. We study the impact of the chosen optimal traffic signal plan on all the routes interconnecting with $\mathrm{C} 129$ in NGC in terms of average travel times and throughput. The last section of the article presents the conclusions and future perspectives of this work.

\section{Related works}

80 2.1. Traffic simulation tools

Numerical simulation is a powerful tool to reproduce real life situations. It can be used to generate, analyse and predict potential problems which are difficult to detect through real tests, which imply high costs, administrative rules to respect, or high risk of incidents. Various urban traffic simulation tools

${ }_{85}$ have been designed according to either the scale of the application (networks, links, intersections), the representation of the process (deterministic, stochastic) or the scale of independent variables (continuous, discrete). But one of the most popular classification criterion is the level of details or more explicitly, the level of description of traffic entities [11. A comparison study between different

90 simulation models depending on the level of details (micro, meso, macro) can be found in 12 . In the remainder of this section, we briefly present the main simulation tools available for each level of detail.

Microscopic traffic simulators have been conceived to simulate at a high level of precision the behaviour of traffic entities inside a transportation system, 95 such as drivers or vehicles, and their interaction with the vicinity. They often require more computational resources and are most suited for simulating small urban areas as they calculate at each time step the speed, position and acceleration of each entity, by applying detailed lane changing and car-following behavioural models. Some of the most popular micro-simulators are: Corsim 100 [13], Vissim [14, Paramics [15, DynaSim [16] and Aimsun [17. A more recent and technical comparative analysis between some of the above mentioned microsimulators can be found in 18 .

In comparison to the above simulation tools, the need to represent traffic at a higher level of aggregation, led to the apparition of macroscopic simulators, 105 such as METANET [19], the cell transmission model [20] or METACOR [21]. These models use an aggregate representation of traffic and are mostly used for offline traffic planning applications. Main drawbacks of these models are related to their restricted ability to model physical queues, spill-backs or route choice decisions. Various macroscopic simulation tools are detailed in [22]. 
At an intermediate level between the micro and macro simulation tools, a special interest is given to mesoscopic traffic simulators. In contrast to the above simulators, at the mesoscopic level, the behaviour of small groups of entities moving together in a probabilistic manner is more important than the individual behaviour of each entity moving in the simulation. The main 115 advantage of mesoscopic models is a solid trade-off between the level of detail and running efficiency. Compared to the macroscopic approach, in a mesoscopic simulation model, the average travel time on a specific road segment is influenced by the segment flow, occupation or its capacity. As well, the supply models in meso-models are simplified compared to microscopic simulation models where individual vehicle movement is approximated by speed-density models on the link, or queue models at the end of a link. Some of the most popular mesoscopic traffic simulators are: DynaMIT [23, DYNASMART 24, Metropolis [25, MEZZO [26, PTV Vissim [27. Most recently, various traffic simulation tools provide hybrid simulation models which offer flexibility to model and switch between the meso and micro-models, such as Aimsun or Vissim.

Also, various urban traffic simulation models have been developed using agent-based models (ABMs). Instead of utilizing traffic flows to represent mobility as the previous mentioned tools, the agent-based modelling represents 130 every traffic participant as an agent which can be assigned specific time schedules or specific activities. ABMs have been successfully used in collaborative driving 48, agent-based driver behaviour modelling [50, route choice analysis [53] and multi-agent based microscopic traffic simulation [51, 52. An overview of potential and existing applications of agent-oriented techniques in transportation modelling can be found in [46. Despite their high popularity for deploying microsimulation models due to a high level of granularity being modelled, ABMs may need more intensive computational capacity when used at larger scales such as macroscopic or mesoscopic.

For the study presented in this paper we use FlexSim 28] for building the ${ }_{140} 3 \mathrm{D}$ traffic simulation models, which offers the possibility to: a) test simultaneously various traffic reconfiguration scenarios in NGC at a mesoscopic level, b) obtain a comparative analysis between scenarios at the end of the simulation, and c) dynamically visualize statistics during simulation. The simulation model is initialised and validated using data provided by the local traffic management 145 centre (TMC), while the simulation output is used as entrance in our evolutionary optimisation module for selecting the best traffic signal plan to be used during rush hours, as detailed in Section 3 .

\subsection{Simulation-based traffic optimization}

The combination of simulation and optimisation techniques has been suc150 cessfully applied to address real-world traffic problems and to assist the decision making process into finding the optimal parameter values for traffic control management. This process often involves a trade-off between satisfying multiple conflicting objectives [29. In a simulation-optimisation approach, the optimisation works as a search method that explores the solution space in such a way 
that solutions leading to the preferred system performances can be found. This approach can be successfully applied for optimizing the traffic signal plans during rush hours, and to simulate the impact of the chosen optimal plan on the traffic system. Although this can be seen as a non-linear programming problem, global optimal solutions are hard to find by traditional mathematical methods.

Evolutionary algorithms (EAs) have become popular for traffic optimization problems, as they are suitable to solve problems that require simultaneous optimisation of more than one objective by using stochastic operators without gradient information in the search process. In the literature, evolutionary or genetic algorithms combined with traffic simulations can be found in 30, 31. In

16532 the authors apply a genetic algorithm optimization and cellular automata in a micro-simulation model from Santa Cruz de Tenerife. Multi-objective genetic algorithms (GAs) have also been used for vehicle scheduling of urban bus lines [33, by improving a non-dominated sorting genetic algorithm (NSGA-II). In 34] the authors used a genetic algorithm with elitism (GAWE) for studying the transit network design problem related to public transportation. The authors in [35] used genetic algorithms to construct and improve the travel time prediction intervals for buses. Multi-objective optimization using GAs has also been successfully applied for road diet plans within transportation networks in [36], or to reduce vehicle emissions, fuel consumption and vehicle delay simultaneously, 175 in an urban intersection from China 37.

Evolutionary and genetic optimization procedures have the advantage of using more than one solution in one iteration (they run on a population approach), unlike most classical optimization algorithms, which update only one solution at each iteration [38. EAs have a global search capability and can effectively balance the solution quality and computational time for complex organisational problems [39. Various studies have shown that EAs are indicated for multi-objective optimization problems, as the population-base meta-heuristic can produce a set of Pareto solutions in a single run [40. In this paper we therefore propose a simulation-optimisation approach at a mesoscopic level by using evolutionary algorithms for the optimization of various traffic signal plans of the intersection C129 (see Section 3.2).

\section{Our proposed optimization framework}

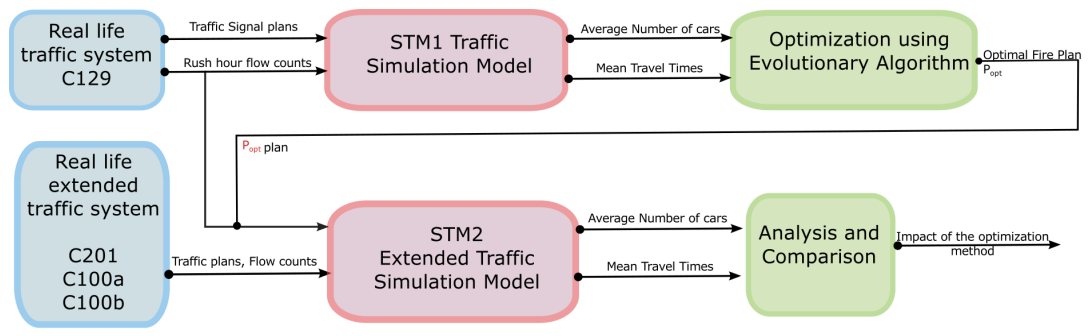

Figure 2: Traffic simulation and optimization framework 
Figure 2 presents the organizational framework of this paper, which contains the traffic simulation models, as well as the evolutionary optimization procedure. We start by analysing the traffic intersection C129, and the available data sets received from Grand Nancy Metropolis, which uses inductive-loop traffic detectors placed in the roadbed at every traffic intersection. The detectors trace vehicles as they pass through the loop's magnetic field and count the number of vehicles during a 60 seconds time interval. Traffic-flow measurements are then

195 stored in a centralised database for further analysis and evaluation. For building the 3D traffic simulation model in FlexSim (denoted as STM1), we use the detailed geometry of the intersection, the available traffic signal plans, the initial OD matrix and registered flow counts during rush hours. During the traffic simulation, vehicles are generated at the main entrances of the intersection, and disposed once they leave the network.

The FlexSim simulation model can output various parameters, including: the mean number of cars (average flow) passing the intersection/each road section, as well as the average travel-time inside the intersection. These parameters represent the optimisation criteria which is evaluated using the evolutionary 205 algorithm we present in the following section. The main objectives for STM1 are: 1) decreasing the average travel-time during rush hours and 2) increasing the number of vehicles transiting the intersection every day. The C129 intersection is part of a reconfiguration plan of the NGC eco-neighbourhood, therefore needs to absorb a higher inflow of vehicles in the future. The current simulation210 optimisation approach allows us to compare all the available traffic signal plans received from Grand Nancy $\left(P_{55}, P_{70}, P_{80}, P_{90}\right)$, and choose the most adapted plan for rush hours which we denote $P_{o p t}$ (the optimal signal plan). As an example, $P_{55}$ stands for the traffic signal plan wherein the switches between green, yellow and red times, last for a cycle of 55 seconds.

As the eco-neighbourhood will be restructured (new bus lines, lane changes) based on the current traffic flow, this paper focuses on analysing the impact of the optimal traffic signal plan of C129, on three other intersections which are currently interconnected with C129. Using metered data received from Grand Nancy, we build an extended traffic simulation model (denoted STM2) which

220 contains four interconnected crossroads (C129, C201, C100a, C100b), as shown in Figure 2. Various scenarios are considered for the extended simulation and in Section 4.3 we present the impact of applying an optimal signal plan in C129 on the surrounding intersections.

\subsection{Dominance concept and Pareto domain}

225 Prior to discussing the method for obtaining the most suited signal plan, we begin by giving a short description of the dominance concept. Before the decision makers choose a good compromise solution by considering a trade-off between the competing criteria, the search domain can be significantly reduced by taking into consideration only those solutions that would be potential can-

230 didates for the optimal solution [41. The final set which is called the Pareto Domain, is a collection of solutions taken from the total set of solutions which are not being dominated by any other solution within this set. We can say that 
a member of a set is dominated by another one if its values on all the optimization criteria are worse than those of the second member. We divide the solution set in two groups: a set of dominant points and a set of points which are dominated by at least one other solution.

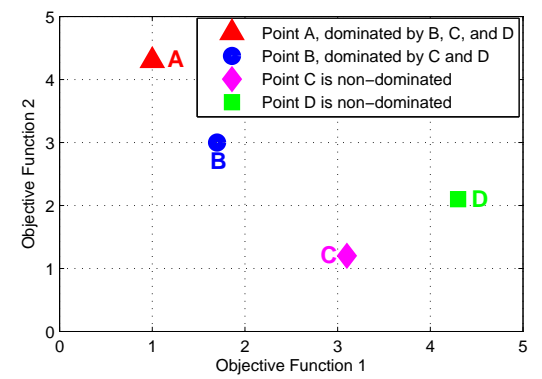

Figure 3: Example of dominance for the Pareto domain.

Figure 3 shows the dominance concept using an illustrative example with two output functions $f_{1}$ and $f_{2}$, which depend on two input variables $x_{1}$ and $x_{2}$. In this example, we want to maximize the objective function $f_{1}$ and minimize $f_{2}$. Point $A$ has the highest value for $f_{2}$ and the lowest value for $f_{1}$, therefore we say that $A$ is dominated by all the other points $B, C$ and $D$, and it cannot be considered as optimal. Although point $B$ has lower values than $A$ in terms of $f_{2}$, it is still dominated by $C$ and $D$ in terms of $f_{1}$. Comparing $C$ and $D$, we observe that both points have better values in one function than the other, therefore 245 we call them non-dominated points which would belong to the Pareto domain if there would not be other generated points that meet the above criteria.

\subsection{The evolutionary algorithm}

The current evolutionary algorithm (EA) is a population-based stochastic search procedure which selects the best members of a population and uses them to recombine and perturb locally, in order to create subsequent populations until a predefined goal was reached. Our approach is based on the evolutionary algorithm described in 42, which we adapt and implement for our transportation problem in NGC. This EA has been chosen due to a good compromise between execution times and computational precision during initial testing of the multiobjective optimisation method. Due to the scope of this paper, we focus more on the result interpretation and impact analysis rather than a comparison of various EA approaches that could be applied for solving the current problem. Algorithm 1 gives the outline of our developed algorithm. We further detail its components.

260 1. The current EA is an iterative optimization process starting from an initial population with nind individuals (points), which are supplied by the traffic simulation model (as represented in Figure 2), and which are characterized by the mean number of cars and the average travel-time inside the intersection. Intersection C129 has six interconnected streets. Let 
$N r_{\text {cars }}^{i}$ be the mean number of cars on the $i^{t h}$ street, $i \in\{1, . .6\}$, and $T_{\text {avg }}^{i}$ the average travel-time on each street $i \in\{1, . .6\}$. The initial number of individuals nind also represents the initial number of solutions (exemplified in Section 4.1.4).

The function initialise_population is responsible for the initialization of all individuals in the first generation. $P^{(n g e n)}$ denotes the entire population of individuals, constructed in the $n g e n^{\text {th }}$ generation. At this step, each point of the initial population has a domination score of 0 .

2. The next step is the computation of two objective criteria for each individual, using the function calculate_objective_fct: maximize the number of cars inside the C129 and minimize the average travel-time.

3. Based on the results obtained for the objective criteria, a fitness function is computed for each individual, using its domination score. Every pair of individuals is compared and, if one dominates the other, the domination score of the latter is incremented by one. The entire solution set is then ordered from best to worst, according to the domination score. A new set of solutions (the new generation) is further constructed in function calculate_nr_dominants by keeping: all the dominant points (those having a domination score of 0 ) and a pre-determined percentage of the dominated points having the lowest domination number. The number of the new survivals nsurv is then computed as:

$$
n s u r v=n r d o m+F L O O R\left(t_{s}(\text { nind }- \text { nrdom })\right),
$$

where $n r d o m$ is the number of dominant points, nind is the initial number of individuals representing the original set, $t_{s}$ is the survival fraction which is randomly chosen $\left(t_{s} \in[0,1]\right)$, and $\operatorname{INT}(a)$ is the integer value of the variable $a$. This subset of nsurv points will be part of the next generation of the Pareto domain. All the above steps are computed in the function select_best_indiv.

4. At this step, mutant solutions are randomly generated in order to increase diversity amongst the population of best survivors, by using the function generate_mutant. Generated mutants will replace current individuals in the population only if they are better than the last survivor.

5. The main part of the algorithm is to replace the dominated points which have been previously eliminated, by new points nominated as "children". The children are the result of combining two random parents $\left(I_{p 1}\right.$ and $\left.I_{p 2}\right)$ from the survivor population, inside the function create_child, according to the equation:

$$
I_{\text {child }}=D_{p} I_{p 1}+\left(1-D_{p}\right) I_{p 2},
$$

where $D_{p}$ is a randomly selected real number between 0 and 1 , each time an input $I_{\text {child }}$ will be created. This step will generate a new individual for the final optimal solution set, replacing its eliminated ancestor.

6. Steps (2) to (5) are repeated until at least $90 \%$ of the generated solution set points are dominant points. The final set is assumed to approximate 
the true Pareto domain, which provides the optimal information to the decision experts, and all solutions outside the Pareto should be avoided for further interpretation. In the present article we apply the EA for every traffic signal plan we study, and conduct a detailed comparison of the results in Section 4.1.4.

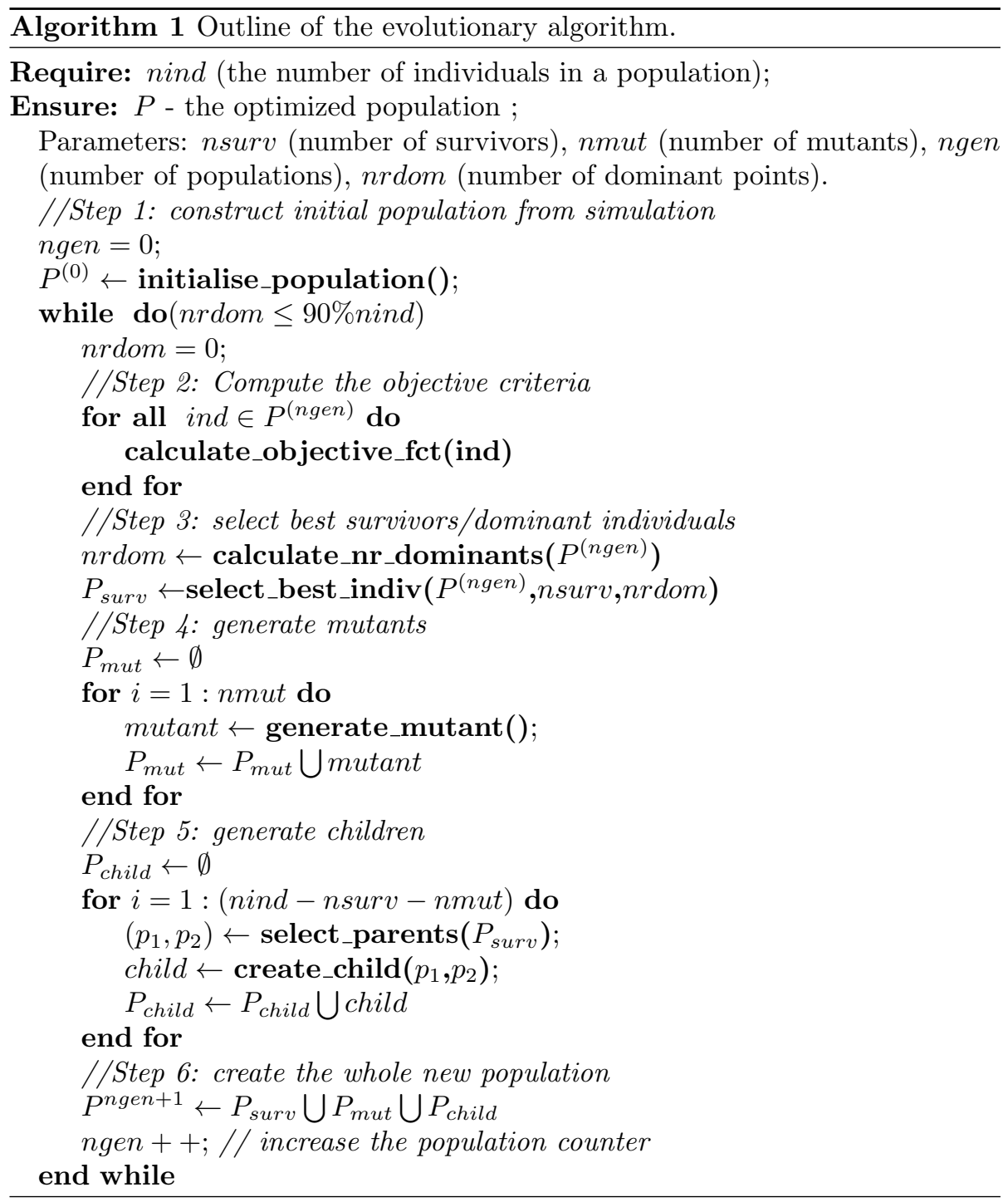




\section{CASE STUDY}

Our case study is a real case optimization project for Metropolis' engineers. 300 They have to efficiently manage the urban transformation from a 15-hectares neighbourhood to an eco-neighbourhood. The current area does not contain housing or retail, and the location and width of the railway right-of-way between C129 and C201 reduce pedestrian presence and walking movements. Furthermore, at the beginning of this study, researchers and local authorities decided to focus only on traffic optimisation aspect. The traffic flow inside NGC is highly affected by the C129 intersection, which suffered various changes in its bus lanes and link connections. Therefore, for Grand Nancy, the main objectives are: a) maximizing the future vehicle inflow in $\mathrm{C} 129$, and b) reducing the average travel-time during rush hours. The case study presented in this section 310 responds to this need by building a 3D mesoscopic simulation and evolutionary optimisation method firstly for C129 (STM1), and secondly for including three other interconnected intersections: C201, C100a and C100b (STM2) and analyse the impact that the local traffic signal optimization in C129 has on the global system.

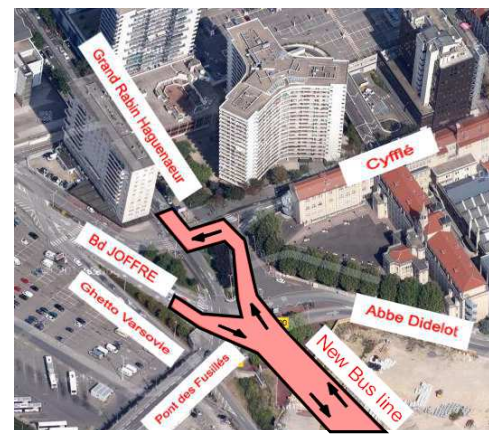

Figure 4: Aerial view of C129 intersection in Nancy, France (Google Maps source) with new bus line added.

\subsection{C129 traffic simulation model (STM1)}

Figure 4 presents an aerial view of $\mathrm{C} 129$ which has 5 entrances: a) Pont des Fusillés bridge (PF), which is the main artery collecting vehicles from south of NGC, b) Joffre Boulevard coming from the train station, c) Ghetto Varsovie street passing under the bridge, d) Grand Rabin Haguenaeur (GRH) street, e)

320 Cyfflé road and $\mathrm{f}$ ) a new lane reserved only for buses. The main roads for vehicles to exit the C129 junction are: Abbé Didelot, Cyfflé and Joffre Boulevard.

\subsubsection{Model construction}

In order to construct the 3D mesoscopic traffic simulation model of $\mathrm{C} 129$, urban planners from Grand Nancy provided: a) the detailed intersection geometry and lane characteristics needed to build the road base and 3D environment of the model, b) the origin to destination matrix, c) detector flow counts every 
15 minutes during rush hours (07:30 - 09:00, 16:30 - 18:00) for 4 months, and d) the traffic signal plans which need to be tested. By traffic signal plan we denote the red-yellow-green cycles of all the traffic lights inside C129, as shown in Figure 5. For example, the traffic light $F 1$ has a starting green-light at second 37 and ends at second 13 in the 55-second cycle length, therefore lasting for 31 seconds. The yellow light lasts for only 3 seconds while the red-light will start at second 17 and end at second 37 after which the green light will be activated again. As an observation, each of the traffic plans are conceived for all normal 335 traffic lights but also pedestrian lights inside the considered intersection. Four signal plans have been received $\left(N_{p}=4\right)$, which we denote: $P_{55}, P_{70}, P_{80}, P_{90}$, each lasting respectively $55,70,80$ and 90 seconds. Due to privacy restrictions from the local community we only showcase the analysis of 4 traffic light plans. Nevertheless the current method can be generalized for various traffic signal plans.

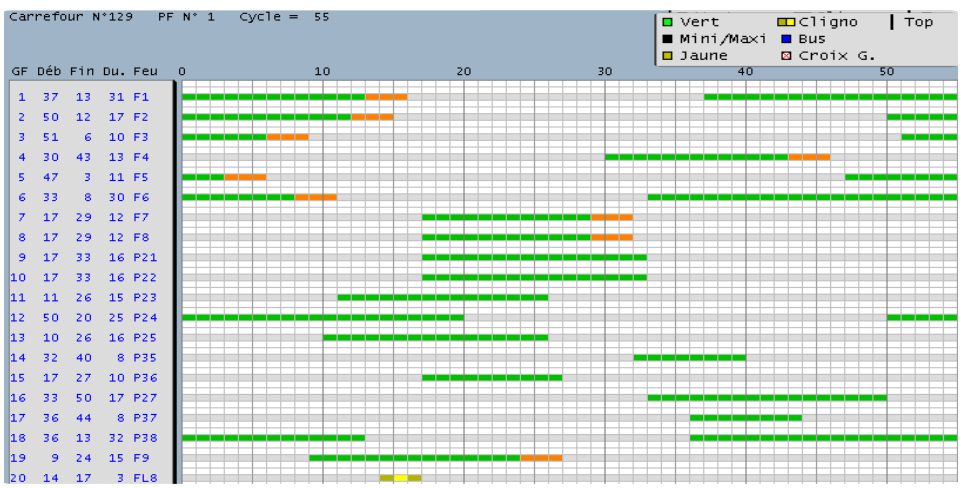

Figure 5: Example of a traffic signal plan $\left(P_{55}\right)$ for C129.

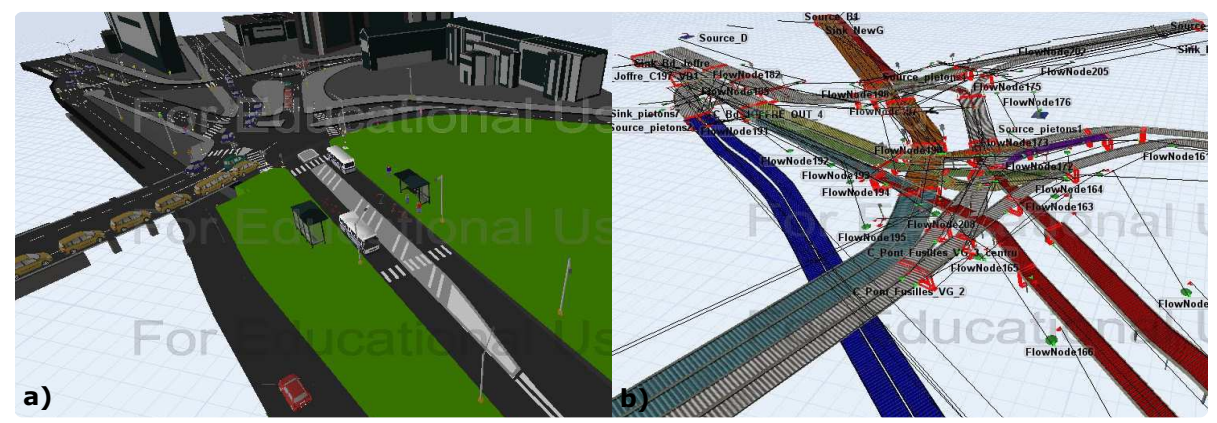

Figure 6: FlexSim 3D simulation model of C129 (a), and its functional structure (b).

A snapshot of the 3D traffic simulation model can be observed in Figure 6a). In Figure 6b) we represent the links between the different simulation objects which need to correspond to the real-life traffic behaviour: conveyors for streets, sources and sinks for generating and disposing the simulated entities (vehicles) in the model, processors for random insertion of vehicles, FlowBin items for pedestrians, Visual Tools for traffic lights which need to correspond 
to the available traffic signal plans. A representation of pedestrian movement around traffic lights and public transport stops is provided in Figure 7 a) and b) respectively. Separate traffic control plans for pedestrian movement have been implemented in the simulation according to the information received from TMC. Although each pedestrian traffic plan corresponds to each of the traffic control plans, the phase times cannot be changed or triggered on demand by pedestrians waiting to cross the intersection.

a)

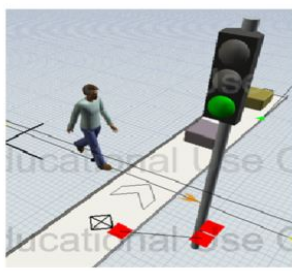

b)

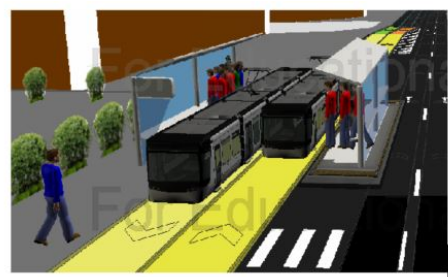

Figure 7: Pedestrians around: a) traffic light plans and b) tramway stops.

The detector flow data has been stored in a dedicated database and the first 2 months were used for initialising the simulation model. The static calibration and validation of the simulation model has been done by using the provided flow counts for the last 2 months and comparing every 15 minutes with real-observed counts registered manually during the morning and afternoon rush hour. The final validated model containing a realistic representation of the traffic in C129 is then used for applying the traffic signal plan optimisation detailed in Section 4.1.4.

\subsubsection{Simulation computing times}

Due to the stochastic behaviour of the simulation model (which needs a large number of replications to be performed), and the complexity of all inter-

365 connected objects, in this section we test the scalability and performance of the simulation model in terms of computing times when a larger number of traffic scenarios need to be tested in parallel or the number of vehicles entering the intersection increases. We consider the following three parameters which have a high impact on the computing time (denoted $T_{c}$ ): the number of replications $\left(N_{r}\right)$, the number of traffic signal plans $\left(N_{p}\right)$, and the number of scenarios to be tested $\left(N_{s}\right)$. More specifically, we fix two of the above parameters, and variate the third one. We further analyse the evolution of $T_{c}$ versus the evolution of the third varying parameter, as shown in Tables 1 to 3 .

The main scenarios tested here come from the need to know the maximal ca-

375 pacity of the intersection in terms of vehicle inflow. Let $D_{2}$ be the total number of vehicles entering C129 (actual metered data received from the municipality). We then construct three additional scenarios based on $D_{2}$, having: $D_{1}=D_{2} / 2$, $D_{3}=D_{2} \times 2$, and $D_{4}=D_{2} \times 3$ vehicles. Let $N_{s}$ be the total number of scenarios which takes values in $[1,4]$; for example the first scenario uses $D_{1}$ vehicles, the second scenario uses $D_{2}$, etc. Finally, as we want to analyse the four available traffic signal plans $\left(P_{55}, P_{70}, P_{90}, P_{80}\right)$, let $N_{p}$ variable to take values in $[1,4]$. 


\begin{tabular}{llllll}
\hline$N_{r}$ & 1 & 5 & 10 & 15 & 20 \\
$T_{c}[$ sec $]$ & 25 & 126 & 271 & 397 & 544 \\
\hline \multicolumn{5}{c}{ Table 1: $T_{c}$ versus $N_{r}}$. \\
\hline$N_{s}$ & $1\left(D_{1}\right)$ & $2\left(D_{2}\right)$ & $3\left(D_{3}\right)$ & $4\left(D_{4}\right)$ \\
$T_{c}[$ sec $]$ & 172 & 353 & 375 & 575 \\
\hline
\end{tabular}

Table 2: $T_{c}$ versus $N_{s}$.

\begin{tabular}{lllll}
\hline$N_{p}$ & 1 (Plan) & 2 (Plans) & 3 (Plans) & 4 (Plans) \\
$T_{c}[\mathrm{sec}]$ & 89 & 185 & 298 & 397 \\
\hline
\end{tabular}

Table 3: $T_{c}$ versus $N_{p}$.

Firstly, we first fix the number of plans and scenarios to: $N_{p}=4, N_{s}=4$, and vary the number of replications $N_{r} \in\{1,5,10,15,20\}$. Table 1 shows the results of $T c$ versus $N_{r}$. Secondly, we fix $N_{r}=15, N_{p}=4$, and vary the number of scenarios $N_{s} \in\{1,2,3,4\}$ (results provided in Table 2). Thirdly, we test the computing times by fixing $N_{s}=4, N_{r}=15$, and vary $N_{p} \in[1,4]$ (see Table 3).

As an observation, the STM1 simulation model is capable of simulating bigger traffic inflows without over increasing the computing time in all of the above cases. The normalized results of the above tests are also represented 390 in Figure 8, and show an empirical analysis of the temporal complexity of $T_{c}$, which is linear in $N_{s}, N_{p}, N_{r}$. We notice a continuous evolution of $T_{c}$ with the increase of the amount of vehicles entering the system (due to different scales, the graphics are normalized : every data point of one parameter is divided by the maximum of that parameter's data set).

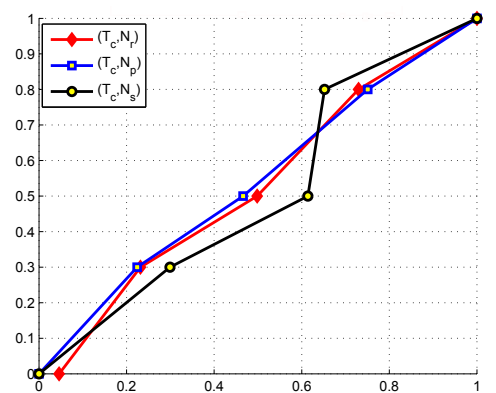

Figure 8: Normalized computing time $T_{c}$ versus $N_{r}, N_{p}, N_{s}$.

The last test has the objective to determine the ideal number of replications which would allow to obtain accurate statistic results, when all the traffic signal plans are tested simultaneously inside the $D_{2}$ scenario, as shown in Table 1 . Following the method presented in [43], we run successive simulations until the average mean and standard variation of the average travel-time (or the mean 400 number of cars) fall within a $95 \%$ confidence interval, using the standard tdistribution. The procedure indicated that running 15 replications for each timeperiod scenario provides accurate results. Increasing this number of replications will not provide any changes in the final statistics. All simulations were executed using an Intel Quad Core i7 $(2.4 \mathrm{GHz})$ computer with 8 GB DDR3 SDRAM memory. 


\subsubsection{STM1 output analysis}

The following simulation outputs are extracted from the STM1 simulation model: the average number of cars during rush hours $\left(N r_{\text {cars }}\right)$ and the average travel-time $\left(T_{\text {avg }}\right)$ in C129. These model outputs represent the two objective criteria that are maximized/minimized using the evolutionary algorithm described in Section 3.2 Other objective criteria can be further added, which might be of a high interest in the optimization process: mean blocking times (or intersection delay), density, occupancy, speed, etc.

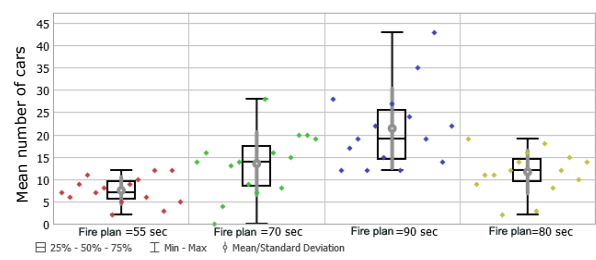

Figure 9: Mean number of cars on Pont des Fusillés, for each traffic signal plans.

Figure 9 shows the variation of the average number of cars on the Pont des Fusillés (PF) street, during morning rush hours, while testing each of the four traffic signal plans inside the $D_{2}$ scenario (real data set). $P_{90}$ signal plan seems to allow a larger number of cars to cross this street and, thus, to be the one suitable for a bigger vehicle inflow. Figure 10 verifies whether this plan is also suitable in terms of average travel-time. Although $P_{90}$ provides as well 420 the smallest travel-time on the PF street (Figure 10 a), it would dramatically increase the travel time on Joffre Boulevard (Figure 10b). This plan would have the same impact on other streets as well, making it difficult to analyse the variation of the mean number of cars and the average travel-time for all the traffic signal plans that need to be tested.

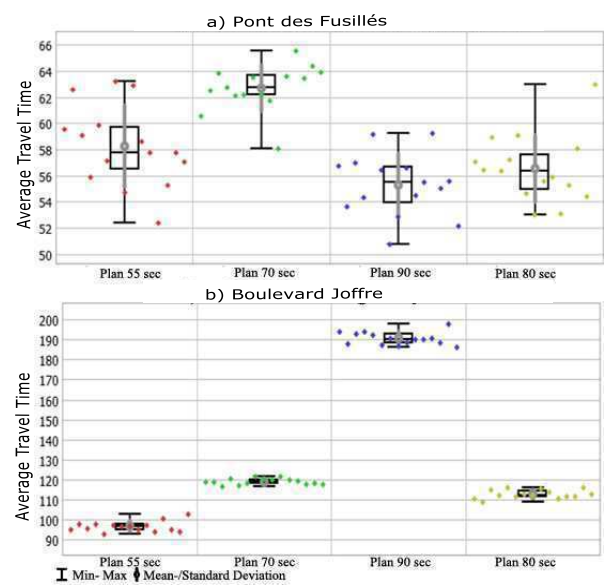

Figure 10: $T_{\text {avg }}$ on a) Pont des Fusillés and b) Joffre Boulevard.

As $\mathrm{C} 129$ will receive a higher vehicle inflow in the future planned reconfiguration of NGC, we test as well the model outputs during the three other scenarios. Figure 11 presents the evolution of the mean number of cars versus the average 
travel time in $\mathrm{C} 129$, through all the four scenarios previously detailed: $\left\{1\left(D_{1}\right)\right.$, $\left.\left.2\left(D_{2}\right), 3\left(D_{3}\right), 4\left(D_{4}\right)\right\}\right)$, and for all the available traffic signal plans $\left\{P_{55}, P_{70}\right.$, $\left.P_{80}, P_{90}\right\}$. For example, the notation $P_{90}-D_{1}$ represents the results obtained when testing the $P_{90}$ plan in the $1^{\text {st }}$ scenario, etc. When analysing the Joffre Boulevard or the Ghetto Varsovie street, there seems to be a clear difference between the results obtained for different traffic plans, indicating that $P_{55}$ might be the most adapted plan to current traffic conditions. But this behaviour does 435 not apply on some other streets where there is a mixture of possible traffic signal plans that might be adapted for higher vehicle inflow. This aspect motivates the application of a multi-objective optimisation criteria through EAs, as further detailed.
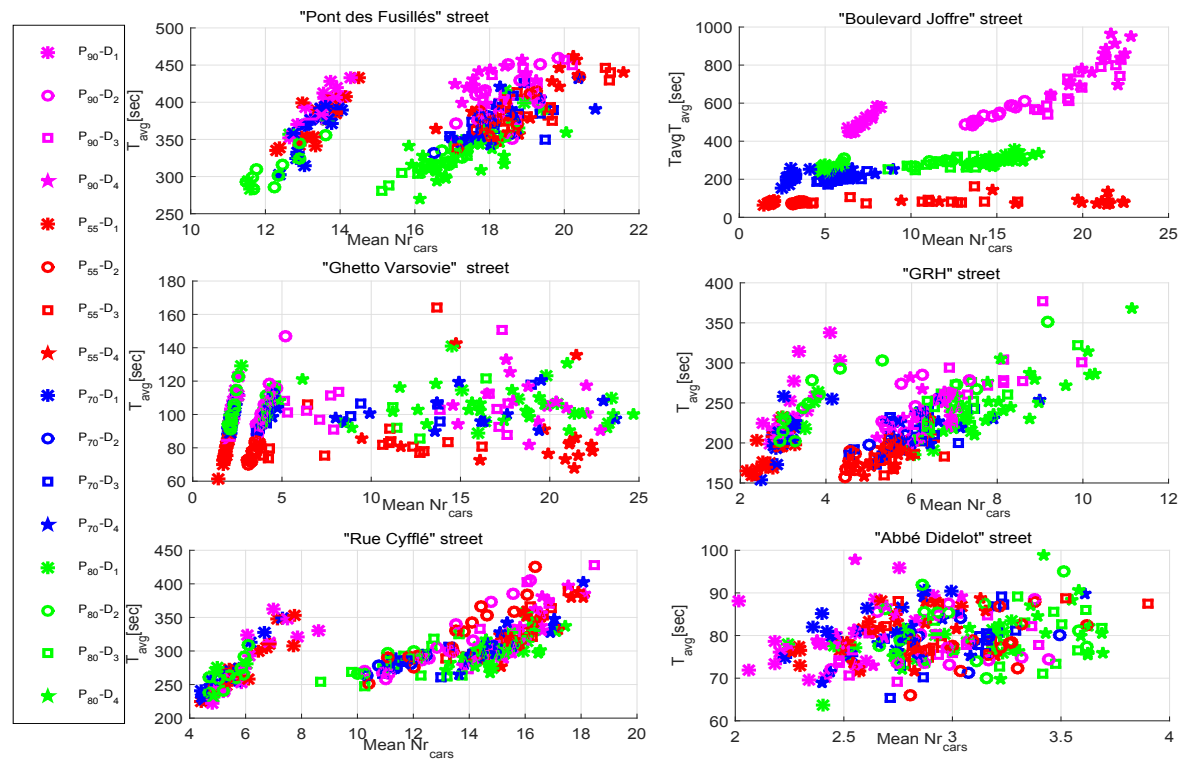

Figure 11: Mean numbers of cars versus average travel-time on each street of C129.

\subsubsection{Optimization problem for STM1}

Previous results confirm the complexity of $\mathrm{C} 129$, for which an adapted optimization method needs to be applied, as detailed in Section 3.2. In our case, we have identified two main criteria by analysing the current needs of TMC. These would translate in finding the best adapted traffic signal plan which will: a) maximize the number of vehicles passing through the intersection during rush hours and: b) minimize the average travel-time spent by these vehicles in the intersection. Therefore, we can express the multi-objective optimization problem as:

$$
\begin{array}{lc}
\text { Maximize } & F_{1}=\sum_{i=1}^{M} N r_{\text {cars }}^{i} \\
\text { Minimize } & F_{2}=\sum_{i=1}^{M} T_{\text {avg }}^{i} \\
\text { subject to } & T_{\text {avg }}^{i} \geq 0 \text { and } N r_{\text {cars }}^{i} \geq 0 .
\end{array}
$$


where $M$ is the number of streets in the intersection, $N r_{\text {cars }}^{i}$ is the average number of cars passing through a street $i$ which belongs to $\mathrm{C} 129$, and $T_{\text {avg }}^{i}$ is the average travel-time spent by vehicles on a street $i \in M$ during rush hours. These criteria will be optimised by applying the evolutionary algorithm previously detailed.

We start by constructing the initial population for the evolutionary algorithm which contains nind individuals and which represents the initial solution of the problem. In our case, nind is the total number of points obtained from each replication of the simulation model (nind $=4$ scenarios $\times 4$ signal plans $\times 15$ replications per scenario). Each point of the population is characterized by the two criteria $F_{1}$ and $F_{2}$ previously defined and which represent the total mean number of cars and the total average travel-time simulated in C129 during rush hours. A representation of the initial population is provided in Figure 12 .

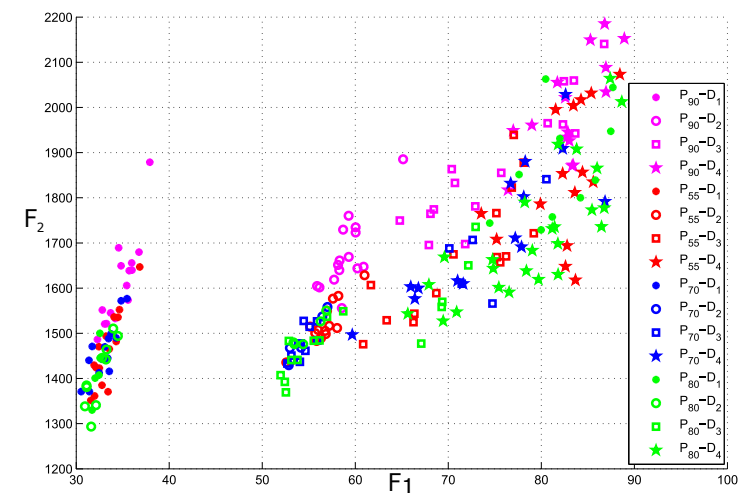

Figure 12: Computation of the $F_{1}$ and $F_{2}$ objective functions for the initial population.

The number of mutants and the survival rate have been set according to the input data $\left(n m u t=5 \%\right.$ nind, $\left.t_{s}=30 \%\right)$. The algorithm is applied until at least $90 \%$ of the final optimal solution contains non-dominated points. During this time, the algorithm creates various generations of individuals until an optimal solution is reached (for our experiments the algorithm gives optimal results after $6-7$ generations).

Figure 13 presents the final optimal solutions of the EA applied for each signal plan. When comparing these solutions, we observe that using the $P_{90}$ plan as an optimal traffic signal plan has a wider spread in solution points than all the other plans, and it also provides the best values for maximizing the number of cars on the streets and minimizing the average travel-time.

475 The observation seems to be even more conclusive when representing only the Pareto front of each traffic signal plan in Figure 14. This result also indicates that any intersection which has reached a saturation point in terms of the accepted vehicle inflow, would produce a high increase in the delay/average travel-time registered in that intersection. Based on the results showed in Figure

480 14. we conclude that $P_{90}$ is the best adapted traffic signal plan for minimizing congestion during rush hours in C129. 

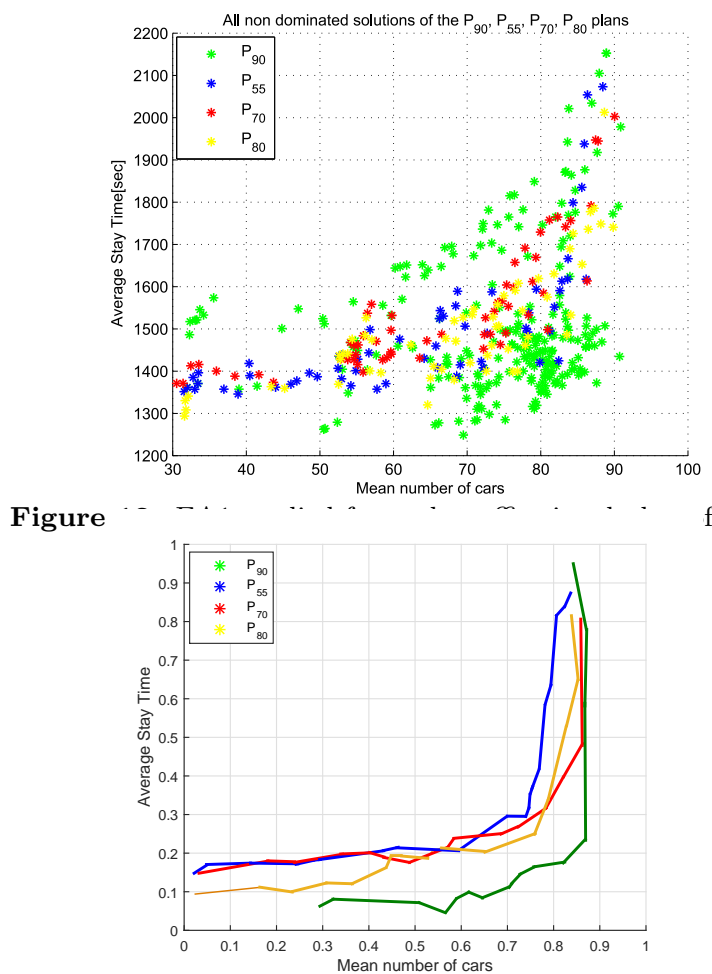

Figure 14: Pareto fronts for each traffic signal plan simulated in STM1.

\subsection{Extended 3D simulation model (STM2)}

The C129 intersection plays a central part in the NGC eco-neighbourhood and the optimization method applied locally will have an important impact on the traffic flow in all the interconnected crossroads. To analyse this impact, the second part of this case study presents the construction of the extended traffic simulation model (STM2), as represented in Figure 2, and which contains four interconnected crossroads: C129, C201, C100a and C100b. Using similar data received from Grand Nancy, we apply the previous steps for building and validating the 3D traffic simulation model. A snapshot of the extended 3D mesoscopic simulation is provided in Figure 15 and a 3D visualisation video can

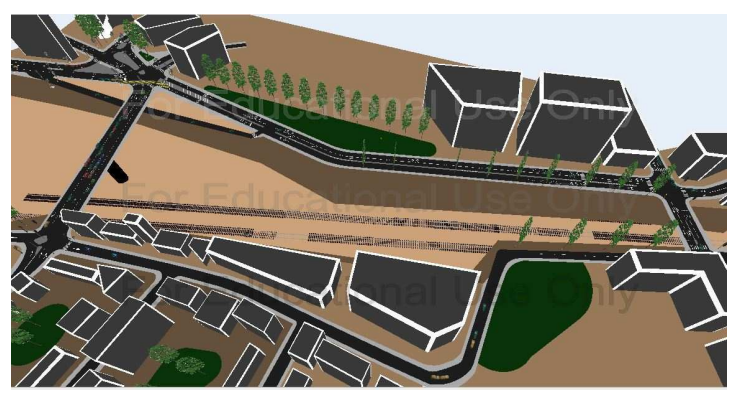

Figure 15: STM2 simulation model in FlexSim. 
be seen in 45. If STM1 has 5 entrances and 4 exits, the extended simulation model STM2 (which lays over the railway tracks) has a total of 8 main entrances (denoted $\left.E_{i}, i \in\{1, . .8\}\right)$ and 8 main exits $\left(S_{j}, j \in\{1, . .8\}\right)$. A graphical representation of STM2, with various ramifications and lane switching from one intersection to another, is provided in Figure 16.

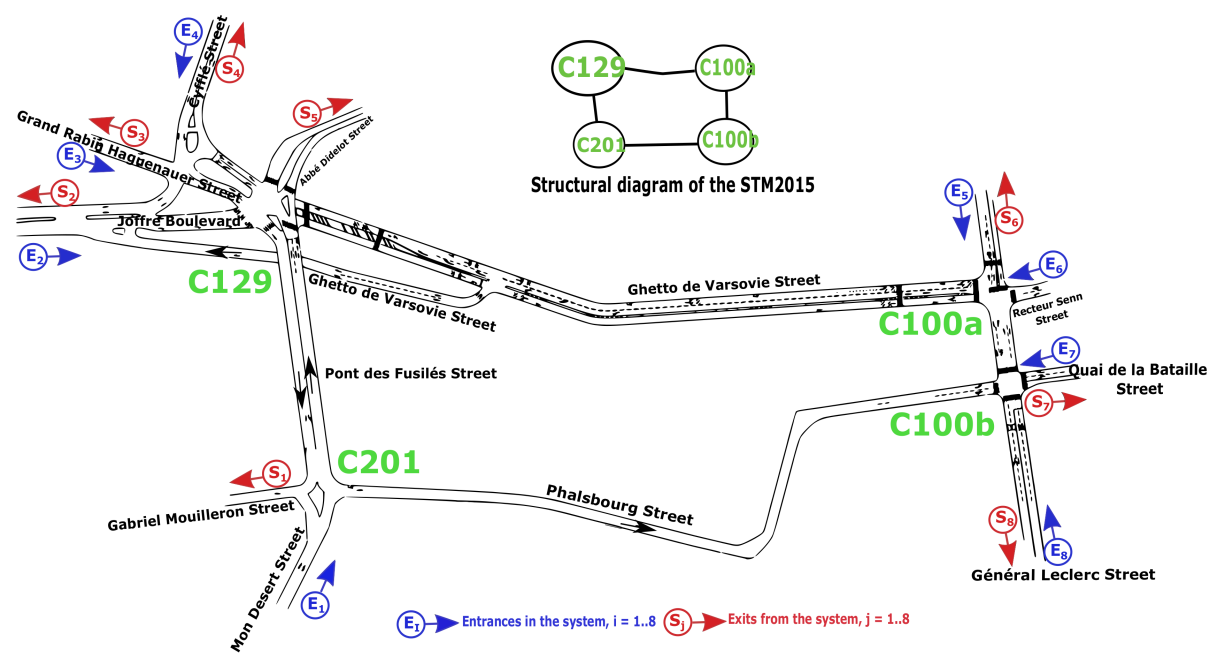

Figure 16: Diagram of the entrances and exits of STM2.

Let $t_{i j}, i, j \in\{1, . .8\}$, be the route starting from an entrance point $E_{i} \in$ $\left\{E_{1}, \ldots E_{8}\right\}$ and ending at an exit point $S_{j} \in\left\{S_{1}, \ldots S_{8}\right\}$. Due to the physical interconnections between the crossroads, from all the 64 origin to destination pairs inside the system, we exclude the returning and the short routes which are irrelevant to this study (examples: $t_{11}, t_{22}, t_{33}$, which are marked in orange in Table 4). We therefore analyse 56 routes in terms of mean number of cars and average travel-time.

\begin{tabular}{|l|c|c|c|c|c|c|c|c|}
\hline$O / D$ & $S_{1}$ & $S_{2}$ & $S_{3}$ & $S_{4}$ & $S_{5}$ & $S_{6}$ & $S_{7}$ & $S_{8}$ \\
\hline$E_{1}$ & $t_{11}$ & $t_{12}$ & $t_{13}$ & $t_{14}$ & $t_{15}$ & $t_{16}$ & $t_{17}$ & $t_{18}$ \\
$E_{2}$ & $t_{21}$ & $t_{22}$ & $t_{23}$ & $t_{24}$ & $t_{25}$ & $t_{26}$ & $t_{27}$ & $t_{28}$ \\
$E_{3}$ & $t_{31}$ & $t_{32}$ & $t_{33}$ & $t_{34}$ & $t_{35}$ & $t_{36}$ & $t_{37}$ & $t_{38}$ \\
$E_{4}$ & $t_{41}$ & $t_{42}$ & $t_{43}$ & $t_{44}$ & $t_{45}$ & $t_{46}$ & $t_{47}$ & $t_{48}$ \\
$E_{5}$ & $t_{51}$ & $t_{52}$ & $t_{53}$ & $t_{54}$ & $t_{55}$ & $t_{56}$ & $t_{57}$ & $t_{58}$ \\
$E_{6}$ & $t_{61}$ & $t_{62}$ & $t_{63}$ & $t_{64}$ & $t_{65}$ & $t_{66}$ & $t_{67}$ & $t_{68}$ \\
$E_{7}$ & $t_{71}$ & $t_{72}$ & $t_{73}$ & $t_{74}$ & $t_{75}$ & $t_{76}$ & $t_{77}$ & $t_{78}$ \\
$E_{8}$ & $t_{81}$ & $t_{82}$ & $t_{83}$ & $t_{84}$ & $t_{85}$ & $t_{86}$ & $t_{87}$ & $t_{88}$ \\
\hline
\end{tabular}

Table 4: All possible routes inside STM2.

Currently Grand Nancy is applying only 90 seconds traffic plans in all the 505 interconnected crossroads (C201, C100a, C100b), which remain unchained during rush hours, a requirement which has been respected for the current study. This procedure is mainly related to the fact that, during morning rush hours 
C129 holds the biggest vehicle inflow and outflow compared to its neighbours (see Table 5), mainly due to the direct connection to the city train station.

\begin{tabular}{|ll|l|l|l|l|}
\hline & & $C 129$ & $C 201$ & $C 100 a$ & $C 100 b$ \\
\hline Total & Inflow & 2765 & 2142 & 1567 & 1729 \\
Total & Outflow & 2786 & 2190 & 1581 & 380 \\
\hline
\end{tabular}

Table 5: Total inflow/outflow inside STM2.

Let $P_{90}-\mathrm{C} 129, P_{90}-\mathrm{C} 201, P_{90}-\mathrm{C} 100 \mathrm{a}$ and $P_{90} \mathrm{C} 100 \mathrm{~b}$ be the 90 seconds traffic signal plans used in respectively C129, C201, C100a and C100b. Similarly, let $P_{55}-\mathrm{C} 129, P_{70}-\mathrm{C} 129$ and $P_{80}-\mathrm{C} 129$ be the other three traffic signal plans used in C129. Using these notations, the following scenarios with associated signal plans are evaluated in STM2, which give a more realistic approximation of the impact that a local optimization procedure can have on a larger and more complex system:

1. $S C_{1}$ applies: $P_{90}-\mathrm{C} 129, P_{90}-\mathrm{C} 201, P_{90}-\mathrm{C} 100 \mathrm{a}, P_{90}-\mathrm{C} 100 \mathrm{~b}$.

2. $S C_{2}$ applies: $P_{80}-\mathrm{C} 129, P_{90}-\mathrm{C} 201, P_{90}-\mathrm{C} 100 \mathrm{a}, P_{90}-\mathrm{C} 100 \mathrm{~b}$.

3. $S C_{3}$ applies: $P_{70}-\mathrm{C} 129, P_{90}-\mathrm{C} 201, P_{90}-\mathrm{C} 100 \mathrm{a}, P_{90}-\mathrm{C} 100 \mathrm{~b}$.

4. $S C_{4}$ applies: $P_{55}-\mathrm{C} 129, P_{90}-\mathrm{C} 201, P_{90}-\mathrm{C} 100 \mathrm{a}, P_{90}-\mathrm{C} 100 \mathrm{~b}$.

\subsection{Impact of C129 optimisation}

Figure 17 presents the average number of cars on all routes in STM2, obtained after simulating all of the above scenarios. Using the 90 -seconds plan in all intersections would maximize the $N r_{\text {cars }}$ on 40 routes, as shown in Table 6 . The most significant impact of applying the $S C_{1}$ scenario can be observed for all the routes starting from Cyfflé street ( $E_{4}$ entrance), which presents almost a $17 \%$ improvement compared to the other scenarios.

\begin{tabular}{|l|l|l|l|l|}
\hline & $S C_{1}$ & $S C_{2}$ & $S C_{3}$ & $S C_{4}$ \\
\hline$E_{1}$ & $t_{12}, t_{16}, t_{18}$ & & $\begin{array}{l}t_{13}, t_{15} \\
t_{17}\end{array}$ & $t_{14}$ \\
\hline$E_{2}$ & $t_{21}, t_{23}, t_{25}, t_{26}, t_{27}, t_{28}$ & & & $t_{24}$ \\
\hline$E_{3}$ & $t_{31}, t_{32}, t_{35}, t_{36}, t_{37}, t_{38}$ & & $t_{34}$ & \\
\hline$E_{4}$ & $t_{41}, t_{42}, t_{43}, t_{45}, t_{46}, t_{47}, t_{48}$ & & & \\
\hline$E_{5}$ & $t_{51}, t_{52}, t_{53}, t_{55}, t_{58}$ & & $t_{54}$ & $t_{57}$ \\
\hline$E_{6}$ & $t_{61}, t_{62}, t_{63}, t_{65}$ & $t_{68}$ & $t_{64}$ & $t_{67}$ \\
\hline$E_{7}$ & $t_{71}, t_{72}, t_{73}, t_{75}, t_{78}$ & $t_{76}$ & $t_{74}$ & \\
\hline$E_{8}$ & $t_{81}, t_{82}, t_{83}, t_{85}$ & & $t_{84}, t_{86}$ & $t_{87}$ \\
\hline
\end{tabular}

Table 6: routes with maximum number of cars for each scenario $S C_{1}, S C_{2}, S C_{3}, S C_{4}$.

Figure 18 presents the results obtained when analysing the average traveltime for all routes of STM2. Observe that in this case is the implementation of the 55-seconds plan in C129 that minimizes the travel-time on 41 routes, as resumed in Table 7 . This can be explained by some of the initial results obtained for Boulevard Joffre or Ghetto Varsovie street, but also due to the fact that a shorter traffic signal plan: a) would assure a minimal travel-time for the vehicles already in the intersection, b) but would not allow a high flow of vehicles 

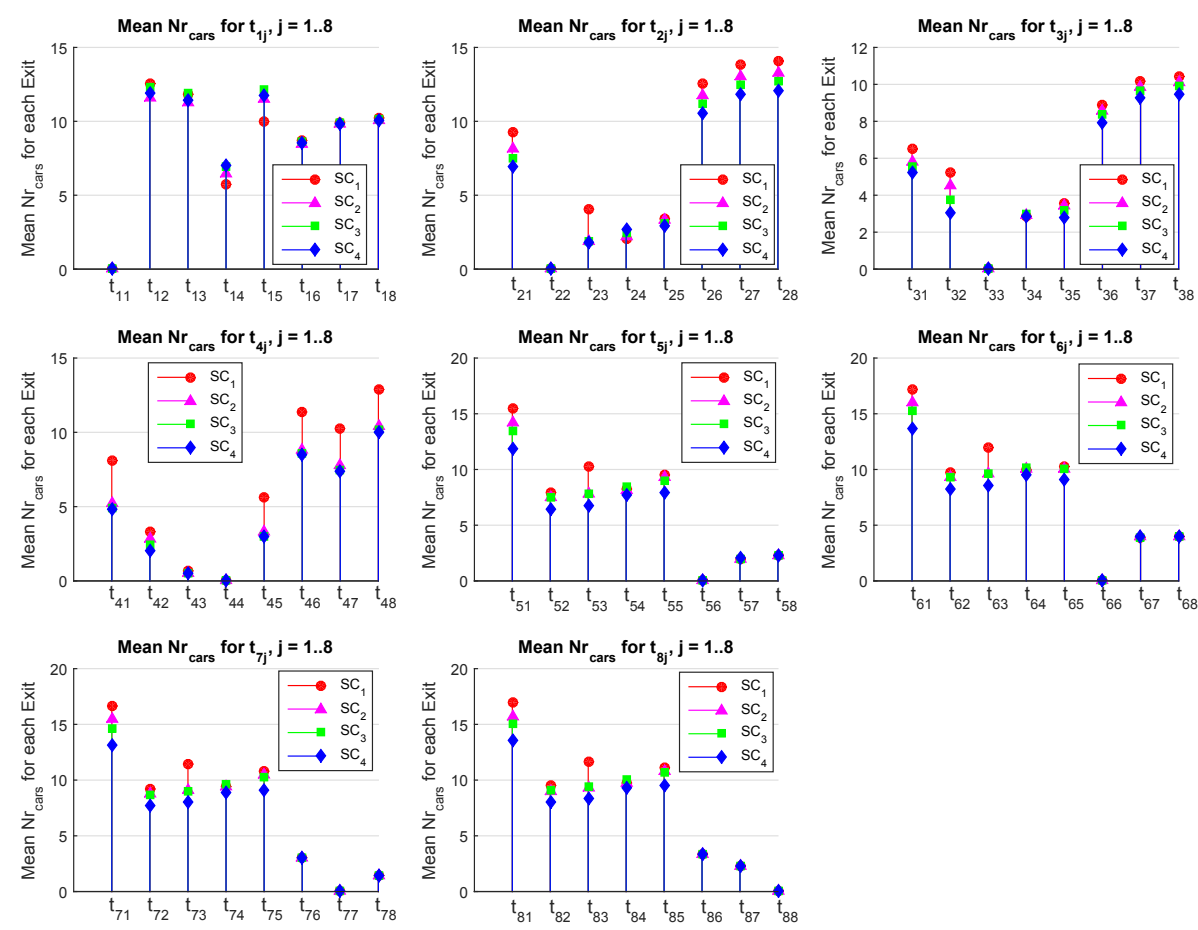

Figure 17: Average number of cars on all the routes of STM2.

to cross the intersection. Interestingly, on small routes which are far away from C129 (such as $t_{67}, t_{68}, t_{76}, t_{78}, t_{86}, t_{87}$ ), switching between traffic signal plans has a low impact; this aspect is available for both mean number of cars and average travel-time. On the other hand, all the routes currently connected to the congested streets of $\mathrm{C} 129$ will be directly affected by the changes of the traffic signal plans, such as $t_{53}, t_{63}, t_{73}, t_{83}$.

\begin{tabular}{|l|l|l|l|l|}
\hline & $S C_{1}$ & $S C_{2}$ & $S C_{3}$ & $S C_{4}$ \\
\hline$E_{1}$ & $t_{14}, t_{15}$ & $t_{12}$ & & $t_{16}$ \\
& $t_{17}, t_{18}$ & $t_{13}$ & & $t_{16}$ \\
\hline$E_{2}$ & $t_{24}$ & & $t_{23}$ & $t_{21}, t_{25}, t_{26}, t_{27}, t_{28}$ \\
\hline$E_{3}$ & $t_{34}$ & & & $t_{31}, t_{32}, t_{35}, t_{36}, t_{37}, t_{38}$ \\
\hline$E_{4}$ & & & & $t_{41}, t_{42}, t_{43}, t_{45}, t_{46}, t_{47}, t_{48}$ \\
\hline$E_{5}$ & & $t_{57}$ & $t_{58}$ & $t_{51}, t_{52}, t_{53}, t_{54}, t_{55}$ \\
\hline$E_{6}$ & $t_{67}, t_{68}$ & & & $t_{61}, t_{62}, t_{63}, t_{64}, t_{65}$ \\
\hline$E_{7}$ & $t_{78}$ & & $t_{76}$ & $t_{71}, t_{72}, t_{73}, t_{74}, t_{75}$ \\
\hline$E_{8}$ & & & & $t_{81}, t_{82}, t_{83}, t_{84}, t_{85}, t_{86}, t_{87}$ \\
\hline
\end{tabular}

Table 7: Routes with minimum travel-time for each scenario $S C_{1}, S C_{2}, S C_{3}, S C_{4}$.

Discussions and method generalisation: Extending the dimensions of the system is an important aspect to be considered, and the results show a future need for studying the impact and the propagation of a local optimization 

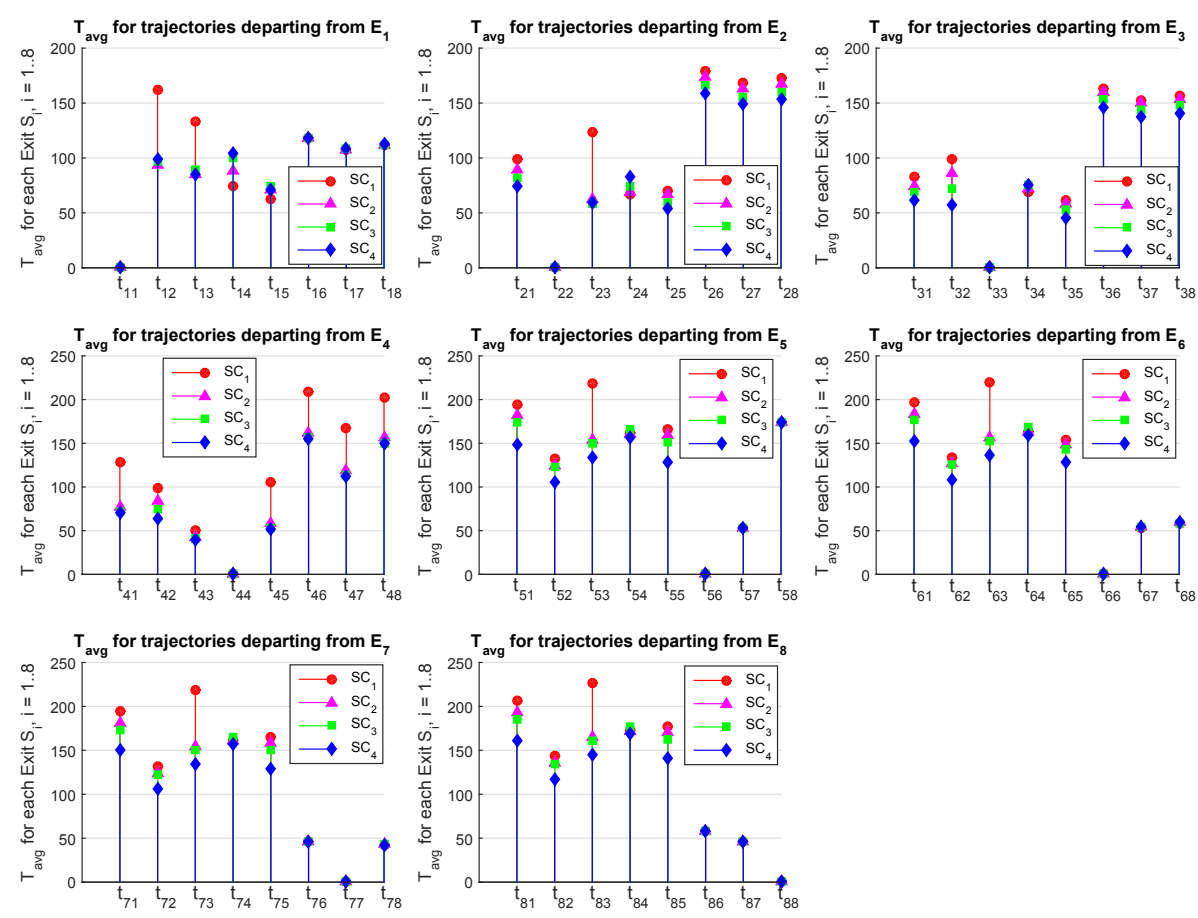

Figure 18: Average travel-time $T_{a v g}[\mathrm{sec}]$ on all the routes of STM2.

method in a larger system. A bigger amount of data would be necessary for testing the behaviour of the system on longer periods of time, while other traffic signal plans can be tested for all intersections of STM2. This aspect would need further approval and collaboration with Grand Nancy. While other methods propose decentralized optimization methods for larger transportation networks, our method (which has been initially created to respond to a real community demand) shows the importance of analysing the impact of local optimization and reconfiguration scenarios in urban environments. For a general approach of our method, independently of the neighbourhood or area to be simulated, the following steps are applied:

1. build the traffic simulation model/s of the interest area (intersection, neighbourhood, etc.).

2. apply a data processing and cleaning methodology to build the origin-todestination matrix, as well as the validation datasets (e.g. counts or travel time when available).

3. calibrate and validate the simulation model/s against collected datasets.

4. run mesoscopic traffic simulation scenarios using different available traffic plans or specific configuration information for intersection management.

5. use simulation output for multi-objective criteria definition.

6. apply evolutionary algorithms for obtaining the Pareto front and finding the best solution that satisfies all the criteria. 
7. choose the optimal solution and re-run either the initial simulation model with new datasets during peak hours or the extended simulation model (multiple intersections, larger areas).

The proposed methodology is also scalable for the increased number of intersections compared to the base model. Our simulation tests showed a compu570 tational time that has doubled for the STM2 compared to STM1 when testing all four traffic light plans, mainly due to an increased number of lanes, detectors and traffic lights that need to simulated. The traffic control plan evaluation is done in parallel in the simulation model which saves computational time and comparison effort.

\section{Conclusions}

This paper proposed an urban traffic multi-objective optimization method for intersections belonging to an transforming neighbourhood from Nancy, France. Initially, we constructed the C129 traffic simulation model in FlexSim, using real-traffic data sets from the local traffic management center, which allowed us to obtain the objective criteria we want to optimize. Using evolutionary algorithms for the optimization process, we obtained the Pareto fronts for all available traffic signal plans which have been simulated.

Results: This method identified P90 as the optimal plan to be adopted in C129 that would accept a higher inflow of vehicles to pass the intersection during rush hours, in a shorter average travel-time. The optimal plan has been tested as well in an extended traffic simulation model containing three other intersections linked to C129. All possible combinations of traffic signal plans have been also tested inside the extended model. The last part of our work showed that adopting the 90-seconds plan in all intersections during rush hours would maximise the number of cars on $71.4 \%$ of all possible routes in NGC, while switching to a 55 -seconds in C129 would minimize the average travel times on $75 \%$ of the routes. As results indicated, a local optimisation in C129 will highly impact traffic conditions in all NGC intersections.

\section{Main contributions:}

On the research side: we proposed a new application of evolutionary algorithm confirming its robustness. Furthermore, an original integrated framework using both 3D mesoscopic traffic simulation and multi-objective optimisation allows the comparative scenario testing to support traffic operations and decision making.

On the operational side: this study allowed developing a tool for public action dedicated to traffic and public transportation management. The first result was the development of an internal Metropolis tool. However, engineers also imagine that this new way of data visualization could help to interact and work with neophytes and users. Further works will be developed to evaluate this useful function for civic participation and knowledge sharing, which are inherent elements of developing an urban eco-neighbourhood. These first outcomes also 
encourage new professional practices orienting engineers towards traffic prediction and mobility forecast for studying usage scenarios and their impacts.

On the technological and pedagogical side: we designed new functionalities and applications for an Assisted Production Management software involving a group of engineering students (M1 level). This planning and decision tool is currently used as a communication and pedagogical tool which allows training future traffic engineers.

Model limitations: The main limitation of the current case study is the number of candidate traffic signal plans, mainly due to limited data provided by the Metropolis traffic authorities. This exploratory study is based on a real traffic counting and the needs of the technical department in Metropolis, in particular for rush-hour situation. In fact, it is a first step and this work will be extended. The volume of data gathered and examined should be increased to study a more complex traffic situation including different traffic flows.

As empirically shown in Table 3 , the simulation time for the mesoscopic model is linear with the number of considered traffic signal plans. By extrapolation, one would expect around 2.75 hours for simulating 100 possible traffic signal plans in C129, proving that simulation time is not a bottleneck of our ${ }_{25}$ proposed framework.

Future work: We are currently engaged with the Metropolis of Grand Nancy for obtaining additional data for our simulation. Therefore, the immedi-

ate perspective of this work is oriented towards testing additional combinations of possible traffic signal plans for various interconnected crossroads in NGC.

As the Nancy Grand Cœur is labelled as an emerging eco-neighbourhood for mobility, the air quality inside this neighbourhood is also very important. We already started collecting data and looking at methods for integrating together traffic simulation models and vehicular emission models, which would help predict the impact of transportation changes on the air quality in the city 635 44]. Ongoing studies are currently analysing the evolution of main vehicle pollutants and traffic behaviour in the Nancy Grand Cœur eco-neighbourhood.

\section{Acknowledgements}

This work has been entirely developed in the ERPI research team from University of Lorraine France, under the Chaire REVES project funding. The 640 authors of this work are grateful for the data and support provided by Grand Nancy and FlexSim Conseil. The final writing and submission of the paper has been done in DATA61 - CSIRO research entity from Sydney, Australia.

\section{References}

[1] UN, World urbanization prospects: The 2014 revision, highlights (st/esa/ser.a/352), Report, United Nations, Department of Economic and Social Affairs, Population Division (2014). 
[2] C. Schweizer, F. Racioppi, L. Nemer, Élaboration de plans nationaux daction pour les transports, la santé et lenvironnement. Manuel pas à pas destiné aux décideurs et planificateurs., Technical report, Europe: Bureau regional de l'organisation mondiale de la santé (2014).

[3] H. van Essen, A. Schroten, M. Otten, External costs of transport in Europe, Technical report, CE Delft (2011).

[4] M. Trilling, Decreasing dependence on fossil fuels: the role of EU public banks in energy financing in central and eastern europe., Technical report, 655 Prague: CEE Bankwatch and Friends of the Earth Europe EU Funds Campaign Coordinator (2013).

[5] M. Ruano, Ecourbanism. sustainable human settlements: 60 case studies, Technical report, Gustavo Gili, Barcelona (1999).

[ [6] Ministère du logement et de l'habitat durable, Les ecoquartiers www. logement.gouv.fr/les-ecoquartiers (2016).

[7] L. Dupont, L. Morel, C. Guidat, Innovative public-private partnership to support smart city: the case of $\{$ Chaire REVES\}, Journal of Strategy and Management 8 (3) (2015) 245-265.

[8] S. Kyvelou, T. Papadopoulos, Exploring a south-european econeighbourhood model: planning forms, constraints of implementation and emerging resilience practices, International Journal of Sustainable Development $14(1 / 2)(2011) 77-94$.

[9] Grand Nancy Metropolis, Nancy Grand Cœur www.grand-nancy.org/ grands-projets/nancy-grand-coeur/(2016).

[10] A. Mihăiţă, M. Camargo, P. Lhoste, Evaluating the impact of the traffic reconfiguration of a complex urban intersection, 10th International Conference on Modelling, Optimization and Simulation (MOSIM 2014), Nancy, France, 5-7 November (2014).

[11] S. P. Hoogendoorn, P. H. Bovy, State-of-the-art of vehicular traffic flow modelling, Proceedings of the Institution of Mechanical Engineers, Part I: Journal of Systems and Control Engineering 215 (4)(2001) 283-303.

[12] D. Ni., Multi-scale modelling of traffic flow, Mathematica Aeterna 1 (1) (2011) 27-54.

[13] Y. Xu, Q.-J. Kong, S. Lin, Y. Liu, Urban traffic flow prediction based on road network model, in: 2012 9th IEEE International Conference on Networking, Sensing and Control (ICNSC), (2012) 334-339.

[14] K. Shaaban, I. Kim, Comparison of simtraffic and VISSIM microscopic traffic simulation tools in modelling roundabouts, Procedia Computer Science, the 6th International Conference on Ambient Systems, Networks and Technologies (ANT-2015) (2015) 43-50. 
[15] N. T. Ratrout, I. Reza, Comparison of optimal signal plans by synchro and transyt-7f using PARAMICS: a case study, Procedia Computer Science, the 5th International Conference on Ambient Systems, Networks and Technologies (ANT-2014) (2014) 372-379.

[16] K. Nishimoto, C. H. Fucatu, I. Q. Masetti, Dynasim - a time domain simulator of anchored fpso, Journal of Offshore Mechanics and Arctic Engineering 124 (4) (2002) 203-211.

[17] I. A. Ntousakis, I. K. Nikolos, M. Papageorgiou, On microscopic modelling of adaptive cruise control systems, Transportation Research Procedia, 4th International Symposium of Transport Simulation (ISTS'14) Selected Proceedings, Ajaccio, France, (2015) 111-127.

[18] D. Sun, L. Zhang, F. Chen, Comparative study on simulation performances of CORSIM and VISSIM for urban street network, Simulation Modelling Practice and Theory 37 (2013) 18-29.

[19] Y. Wang, A. Messmer, M. Papageorgiou, Freeway network simulation and dynamic traffic assignment with metanet tools, Transportation Research Record: Journal of the Transportation Research Board 1776 (2001) 178188.

[20] C. F. Daganzo, The cell transmission model: A dynamic representation of highway traffic consistent with the hydrodynamic theory, Transportation Research Part B: Methodological 28 (4) (1994) 269 - 287.

[21] H. H. Salem, M. Papageorgiou, Metacor : a dynamic macroscopic simulation tool for corridor traffic, CESA 1998-IEEE (1998).

[22] S. Zegeye, B. D. Schutter, J. Hellendoorn, E. Breunesse, A. Hegyi, Integrated macroscopic traffic flow, emission, and fuel consumption model for control purposes, Transportation Research Part C: Emerging Technologies 31 (2013) 158-171.

[23] Y. Wen, Scalability of dynamic traffic assignment, Massachusetts Institute of Technology (2009).

[24] M. Ben-Akiva, M. Bierlaire, Discrete choice methods and their applications to short term travel decisions, in: Handbook of Transportation Science, International Series in Operations Research and Management Science, Vol. 56, (2003) 7-37.

[25] A. d. Palma, F. Marchal, Real cases applications of the fully dynamic METROPOLIS tool-box: An advocacy for large-scale mesoscopic transportation systems, Networks and Spatial Economics 2 (4) (2002) 347-369.

[26] T. Toledo, O. Cats, W. Burghout, H. N. Koutsopoulos, Mesoscopic simulation for transit operations, Transportation Research Part C: Emerging Technologies, special issue on Transportation Simulation Advances in Air Transportation Research, 18 (6), (2010) 896-908. 
[27] Lu Lu, Tianlong Yun, Li Li, Yuelong Su, Danya Yao, A Comparison of Phase Transitions Produced by PARAMICS, TransModeler, and VISSIM, IEEE Intelligent Transportation Systems Magazine 2 (3), (2010) 19-24.

[28] Transport simulation in Flexsim https://www.flexsim.com/fr/ (2016).

[29] R. Halim, M. D. Seck, The simulation-based multi-objective evolutionary optimization (simeon) framework, Proceedings of the 2011 Winter Simulation Conference, (2011) 2834-2846.

[30] S. Anfilets, V. Shuts, The use of natural optimization algorithms for the implementation of adaptive control at the crossroad, in: Proceedings of the 12th International Conference Reliability and Statistics in Transportation and Communication, Riga, Latvia, (2012) 227-233.

[31] L. Zhiyong, Immunity genetic algorithms based adaptive control method for urban network signal, Control Theory \& Applications 23 (1), (2006) $119-125$.

[32] J. Sanchez Medina, M. Galn Moreno, E. Rubio, Evolutionary computation applied to urban traffic optimization, in: W. Kosinski (Ed.), Advances in Evolutionary Algorithms, InTech (2008).

[33] X. Zuo, C. Chen, W. Tan, M. Zhou, Vehicle Scheduling of an Urban Bus Line via an Improved Multi objective Genetic Algorithm, IEEE Transactions on Intelligent Transportation Systems 16 (2), (2015) 1030-1041.

[34] M. Nayeem, M. Rahman, M. Rahman, Transit network design by genetic algorithm with elitism., Transportation Research Part C: Emerging Technologies 46, (2014) 30-45.

[35] A. Khosravi, E. Mazloumi, S. Nahavandi, D. Creighton, J. V. Lint, A genetic algorithm-based method for improving quality of travel time prediction intervals, Transportation Research Part C: Emerging Technologies 19 (6), (2011) 1364-1376.

[36] K. Sohn, Multi-objective optimization of a road diet network design, Transportation Research Part A: Policy and Practice 45 (6), (2011) 499-511.

[37] Z. Zhou, M. Cai, Intersection signal control multi-objective optimization based on genetic algorithm, Journal of Traffic and Transportation Engineering (English Edition) 1 (2) (2014) 153-158.

[38] K. Deb, D. Kalyanmoy, Multi-Objective Optimization Using Evolutionary Algorithms, John Wiley \&amp; Sons, Inc., New York, NY, USA, (2001).

[39] W. Dong, M. Zhou, Gaussian classifier-based evolutionary strategy for multi-modal optimization, IEEE. Trans. Neural Netw. Learn. Systems 25 (6) (2014) 1200-1216. 
[40] A. Konak, D. Coit, A. Smith, Multi-objective optimization using genetic algorithms: A tutorial, Reliab. Eng. Syst. Safety 91 (9), (2006) 992-1007.

[41] A. Vandervoort, J. Thibault, Y. Gupta, An objective-based gradient method for locating the pareto domain, Journal of Chemistry and Chemistry Engineering 5, (2011) 608-623.

[42] J. Thibault, D. Taylor, C. Yanofsky, R. Lanouette, C. Fonteix, K. Zaras, Multi criteria optimization of a high yield pulping process with rough sets, Chemical Engineering Science 58 (1), (2003) 203-213.

[43] J. Archer, K. Högskolan, Indicators for Traffic Safety Assessment and Prediction and Their Application in Micro-simulation Modelling: A Study of Urban and Suburban Intersections, TRITA-INFRA, Royal Institute of Technology, (2005).

[44] A. S. Mihăiţă, M. B. Ortiz, M. Camargo, Integrating a mesoscopic traffic simulation model and a simplified NO2 estimation model for predicting the impact of air pollution, 23rd World Congress on Intelligent Transportation Systems (ITSWC 2016), Melbourne, Australia, ITS-AN-SP0363 (2016).

[45] A. S. Mihăiţăa, 3D simulation of Nancy Grand Cour https://www . youtube.com/watch?v=wSphexsNeEw (2014).

[46] A. Bazzan, F. Klügl, A review on agent-based technology for traffic and transportation. The Knowledge Engineering Review 29 (03) (2014) 375403.

[47] S. Hallé, J. Laumonier, B. Chaib-Draa, A decentralized approach to collaborative driving coordination, in: The 7th International IEEE Conference on Intelligent Transportation Systems, Que, Canada, (2004) 453458.

[48] S. Hallé, B. Chaib-Draa, A collaborative driving system based on multiagent modeling and simulation, Transport. Res. Part C 13 (2005) 320345.

[49] P. Hidas, Modeling lane changing and merging in microscopic traffic simulation, Transport. Res. Part C: Emerg. Technol. 10 (2002) 351371.

[50] P. Hidas, Modelling vehicle interactions in microscopic simulation of merging and weaving, Transport. Res. Part C: Emerg. Technol. 13 (2005) 3762.

[51] A. Doniec, R. Mandiau, S. Piechowiak, S. Espié, A behavioral multiagent model for road traffic simulation, Eng. Appl. Artif. Intell. 21 (2008) 14431454.

[52] R. Mandiau, A. Champion, J.-M. Auberlet, S. Espi, C. Kolski, Behaviour based on decision matrices for a coordination between agents in a urban traffic simulation, Appl. Intell. 28 (2008) 121138.

[53] H. DIA, An agent-based approach to modelling driver route choice behaviour under the influence of real-time information. Transport Research Part C, 10: (2002) 331349. 